\title{
Le réseau d'assainissement parisien en période de crue
}

\author{
Sewerage of Paris in case of flood \\ par O. Jacque \\ Mairie de Paris, section de l'assainissement
}

The Paris sewerage network includes $2,300 \mathrm{~km}$ of galeries, 43 storm overflows and 7 flood plants which protect the town against floods caused by the network. The weaknesses are situated in junction between houses and town network. The five main plants in Paris region, which are the water raising stations of Charenton and Clichy, and the sewerage treatment plants of Achères, Valenton and Colombes, are not totally protected against floods.

\section{LE RÉSEAU D'ASSAINISSEMENT PARISIEN}

Le réseau d'assainissement est composé de 2300 kilomètres de galeries (ovoïdes, collecteurs et ouvrages annexes) qui récoltent les eaux usées et les eaux pluviales.

Ce sont des ouvrages municipaux à l'exception de ceux qui permettent le transit d'eaux provenant de la banlieue qui appartiennent au syndicat interdépartemental de l'agglomération parisienne (SIAAP).

Ils acheminent les eaux vers l'usine d'épuration "Seine Aval ", située à Achères, par l'intermédiaire de quatre grands émissaires. Les volumes journaliers à évacuer sont de l'ordre de 1 million $\mathrm{m}^{3}$ d'eaux usées par temps sec.

Ce réseau a été construit en grande partie au cours de la seconde moitié du siècle dernier. En 1910, l'ensemble de la Ville bénéficiait du " tout à l'égout ".

En période de pluie, les débits peuvent atteindre 5 à 10 fois ceux de temps sec. Le réseau est fréquemment saturé. Afin d'éviter une mise en charge trop importante, 43 déversoirs d'orage permettent de délester le réseau en rejetant les eaux excédentaires dans la Seine.

\subsection{Conséquences d'une crue importante} sur le fonctionnement du réseau

En période de crue, deux types de dysfonctionnement peuvent se présenter :

- l'entrée d'eau en provenance de la Seine dans le réseau,

- l'impossibilité de rejeter les eaux de pluie excédentaires dans la Seine.

De manière à se protéger, le Service de l'Assainissement de Paris a édité un recueil de consignes de crue qui permet de prendre les dispositions nécessaires suivant la montée de la Seine, mesurée actuellement au niveau du pont d'Austerlitz.
Elles entrent en vigueur à la cote 26,70 usuelle Austerlitz. A la cote 31,60 usuelle Austerlitz, tous les déversoirs sont fermés, il n'est plus possible d'évacuer l'excédent d'eau de pluie gravitairement.

Ces consignes consistent essentiellement à :

- isoler les réseaux d'égouts et les galeries souterraines en communication avec le fleuve par des jeux de vannes, des barrages à poutrelles ou des portes à flots,

- rejeter en Seine les eaux de pluie par des postes de crue,

- éviter la submersion des voies publiques par suite d'une montée accidentelle de l'eau dans les égouts à partir des bouches et regards situés dans les zones basses.

Sept usines de crue permettent de se protéger d'inondations provenant du réseau :

$\begin{array}{ll}\text { - MAZAS } & \left(6 \mathrm{~m}^{3} / \mathrm{s}\right) \\ \text { - AUTEUIL } & \left(1 \mathrm{~m}^{3 / s}\right) \\ \text { - CHAMONARD } & \left(1 \mathrm{~m}^{3 / s}\right) \\ \text { - ALMA } & \left(3 \mathrm{~m}^{3 / s}\right) \\ - \text { MONTEBELLO } & \left(1 \mathrm{~m}^{3 / s}\right) \\ \text { - CITE } & \left(1 \mathrm{~m}^{3 / s}\right) \\ \text { - AUSTERLITZ } & \left(1 \mathrm{~m}^{3 / s}\right)\end{array}$

Les trois premières usines relèvent également les eaux de temps sec.

\section{- 1.2 Cas d'une crue sans pluie concomitante}

Le seul risque d'inondation trouvant son origine dans le système d'assainissement est une inondation à partir des bouches d'égout. Deux cas sont possibles.

Tout d'abord, les bouches d'égout sont en relation directe avec le fleuve. Elles doivent être isolées par un murage.

En cas d'inondation à partir de la voie publique par submersion des parapets, il est probable que le fonctionnement du réseau serait rapidement perturbé en cas de pluie (arrêt des usines de crue) et que la majorité des installations seraient 
endommagées. En effet, la quasi totalité des installations mécaniques, électriques et des automatismes sont situés dans des locaux souterrains dont l'étanchéité totale vis-à-vis de la voie publique n'a jamais été recherchée.

Il y aurait, alors, dans les zones inondées, un mélange avec les eaux d'égout. Cela entraînerait une mise en charge du réseau, bien au-delà de ces zones, augmentant ainsi le risque d'inondation des caves des immeubles par l'intermédiaire des branchements particuliers.

\subsection{Cas d'une crue et d'une pluie concomitante}

La probabilité d'une crue et d'une pluie très importante concomitante est très faible. Le système d'assainissement de Paris fonctionne normalement en substituant, lors des crues, les sept usines de pompage aux 43 déversoirs d'orage. Ce n'est qu'exceptionnellement que des inondations très ponctuelles peuvent exister.

Il est, néanmoins, évident que les capacités d'évacuation en Seine de ces sept usines $\left(14 \mathrm{~m}^{3 /} / \mathrm{s}\right)$ est sans rapport avec celles des 43 déversoirs. Il y a un risque de mise en charge du réseau beaucoup plus important. C'est donc l'intensité de la pluie et non la hauteur de la crue qui doit être considérée comme source du risque.

La montée de cheminées autour des ouvrages (bouches d'égout et regards) situés dans les zones les plus basses de Paris permet de protéger la voie publique d'une mise en charge des ouvrages qui dépasserait de quelques décimètres le niveau de la chaussée.

La mise en charge des ouvrages rend particulièrement vulnérables les 92000 branchements particuliers qui relient le système de collecte des eaux, interne à chaque immeuble, et le réseau municipal d'assainissement. Ces branchements dont l'étanchéité laisse fréquemment à désirer, ne résistent pas toujours à la montée des eaux dans les égouts provoquant ainsi l'inondation des caves.

L'inondation des chaussées ne pourrait provenir que de pluies longues et relativement intenses ou de conditions " aval " perturbantes sur le réseau, en particulier l'arrêt de l'usine de Clichy.

Ces inondations ne dureraient que le temps nécessaire à l'évacuation des eaux excédentaires vers les usines d'épuration ou vers le fleuve.

\section{II $\square$ LES INSTALLATIONS DU SYNDICAT INTERDEPARTEMENTAL POUR L'ASSAI- NISSEMENT DE L'AGGLOMERATION PARISIENNE (SIAAP)}

Le Syndicat Interdépartemental pour l'Assainissement de l'Agglomération Parisienne (SIAAP) gère un réseau comportant de très nombreux ouvrages, d'importance très variable, aussi bien dans le domaine du relèvement des eaux, du transport des eaux, de l'épuration... On retiendra, en première approche, les stations de relèvement des eaux de Charenton et de Clichy ainsi que les stations d'épuration d'Achères et de Valenton, la station d'épuration des eaux de Colombes étant en travaux.

\subsection{Usine de Charenton}

L'usine de Charenton a un triple objet :

- relever les eaux d'égout de temps sec des communes de Charenton, Ivry, Alfortville et des communes avoisinantes vers l'émissaire Nord-Est. Ce relèvement des eaux de 3 mètres permet ainsi d'éviter de déverser $200000 \mathrm{~m}^{3}$ d'eaux usées par jour à l'amont immédiat de Paris ;

- dessabler et dégriller les eaux d'égout avant leur entrée dans l'émissaire ;
- lors des orages, les eaux de temps sec continuent à être relevées vers l'émissaire ainsi qu'une partie des eaux de pluie, le surplus des eaux est évacué en Seine et en Marne par des déversoirs d'orages (afin d'éviter des inondations à l'amont de l'usine).

En période de crue de la Seine ou de la Marne, les déversoirs sont progressivement fermés (de 27,85 NGF normal à 29,15 NGF normal pour les réseaux de Charenton et d'Alfortville ; à 29,75 NGF normal pour celui d'Ivry). Les eaux sont encore relevées vers l'émissaire du Nord-Est et évacuées vers la Seine et la Marne

A la cote 31,30 NGF normal, le relevage des eaux vers l'émissaire s'arrête, les eaux étant alors uniquement refoulées vers la Seine et la Marne.

La limite de refoulement des pompes de l'usine est à la cote 35,50 NGF normal environ, soit au-dessus de la crue de 1910 (35,11 NGF normal).

Il y a un risque de mettre en charge les collecteurs à l'amont de l'usine dans les cas suivants :

- impossibilité de fermer les déversoirs d'orage. Cette hypothèse semble très improbable en raison des automatismes de sécurité des barrages des déversoirs. De plus, il faut noter de nombreuses communications entre ce réseau et celui du département du Val de Marne qui comprend de nombreux déversoirs et stations de pompage permettant d'alléger l'ensemble des réseaux en cas de nécessité :

- débit à l'amont de l'usine supérieur à la capacité totale des pompes. La possibilité d'utiliser le relèvement des stations de pompage de l'ensemble du secteur devrait permettre d'éviter toute difficulté ;

- arrêt du fonctionnement des pompes. Les pompes de crue sont alimentées par le réseau EDF ; en cas de panne, il y a la possibilité d'utiliser les moteurs diesel de l'usine, permettant ainsi une autonomie de plusieurs jours.

La protection contre les débordements de la Seine et de la Marne est assurée par des murs pouvant contenir la crue de 1924 augmentée de $0,10 \mathrm{~m}$ ou $0,20 \mathrm{~m}$ selon les endroits, c'està-dire correspondant à des cotes de l'ordre de 33,90 à 34 NGF normal.

En cas de très grande crue, la mise hors service de ces installations entraînerait donc des inondations sur des zones d'habitations importantes ; le risque semble néanmoins très faible en raison des mesures de sécurité prises.

\subsection{Usine de Clichy}

Cette usine a un triple objet :

- relevage des eaux d'alimentation des champs d'épandages par l'aqueduc d'Achères ; cette installation fonctionne essentiellement en été ;

- dessabler le plus possible les eaux avant leur passage en siphon sous la Seine afin de protéger les émissaires ClichyAchères branche d'Argenteuil et Clichy-Achères branche de Bezons ainsi que l'aqueduc d'Achères ;

- usine de crue. Les eaux usées provenant des collecteurs parisiens transitent par l'usine de Clichy pour se diriger par les émissaires vers la station d'épuration d'Achères. Lors des orages, le surplus des eaux, ne pouvant plus passer par les siphons et les émissaires, est envoyé en Seine par un déversoir d'orage situé à l'usine (un débit maximum de $64 \mathrm{~m}^{3} / \mathrm{s}$ a été enregistré le 06/07/87). En temps de crue, le déversoir d'orage est fermé à la cote 27,33 NGF normal à Clichy, correspondant à une hauteur d'eau de 5 mètres à Austerlitz $(30,57$ usuelle Austerlitz environ). Les pompes de crue sont alors mises en service (débit maximum $42 \mathrm{~m}^{3 /} / \mathrm{s}$ ).

Il y a un risque de mettre en charge les collecteurs à l'amont de l'usine, dans les cas suivants :

— impossibilité de fermer le déversoir d'orage (porte à flots) ; 
— débit à l'amont de l'usine supérieur à la capacité totale des pompes et du débit pouvant s'écouler vers Achères ;

- arrêt du fonctionnement des pompes, les moteurs de ces pompes marchant au fuel et à l'électricité.

Le risque essentiel réside dans le fait que, si une pluviométrie très importante survenait alors que la Seine est en crue, pouvant entraîner par là même des infiltrations de la nappe dans les égouts, croissant avec l'importance de la crue, les installations de pompage parisiennes ne pourraient peut-être pas suffire, d'où la crainte d'inonder une grande partie de Paris par mise en charge des collecteurs. Il est difficile d'estimer les superficies qui pourraient être touchées, celles-ci étant fonction de l'importance de la crue et de la pluviométrie.

Mentionnons enfin qu'il y a le risque d'inonder l'usine par arrivée d'eaux à partir de la voie publique à Clichy. En effet, le quai de la Seine, au niveau de l'usine n'est pas protégé contre une crue type 1955. Lors de la crue de 1955, des digues de terre avaient dû être installées le long des quais pour protéger la chaussée.

\subsection{Station d'épuration des eaux d'Achères}

Les risques d'inondations de la station sont essentiellement de 3 sortes :

- inondations par l'aval:

En début de période de crue de la Seine, la station d'épuration peut fonctionner sans difficulté à son débit nominal (2 $100000 \mathrm{~m}^{3}$ par jour soit $24,3 \mathrm{~m}^{3} / \mathrm{s}$ ), l'évacuation des eaux traitées se faisant gravitairement par les canaux de restitution. A partir de la cote 21,03 NGF normal de la Seine à la station, soit 27,80 usuelle environ à Austerlitz (dans l'hypothèse où tous les barrages de navigation sont abattus entre Austerlitz et la station d'épuration), les restitutions ne sont plus possibles gravitairement en raison du niveau du fleuve. Les eaux doivent être relevées par des pompes de crues afin que l'aval ne soit pas noyé. Les installations de pompage sont dimensionnées pour le débit nominal de la station.

- inondations par l'amont:

Par ailleurs, afin de ne pas noyer, en cas d'arrêt des pompes de relevage de l'aval, le prétraitement de l'usine puis ultérieurement, l'ensemble de l'usine par des arrivées d'eau trop importantes, il est nécessaire de pouvoir limiter ou arrêter les débits à l'amont.

Précisons que l'alimentation de la station se fait de la façon suivante : quatre émissaires sont actuellement en service. Le passage de ces ouvrages sous la Seine se fait en siphon. Au site de La Frette, tête amont des siphons, sont installées les vannes de réglage des émissaires ; des déversoirs complètent le système. En crue; afin de limiter les débits, des manœuvres sont effectuées afin de faire monter progressivement les plans d'eau des émissaires, en suivant le niveau de la Seine, de façon à ce qu'ils soient toujours supérieurs à celui du fleuve et permettre ainsi le déversement. Des vannes commandées électriquement commandent ces manœuvres. Quand les déversements ne sont plus possibles, on doit fermer les déversoirs, d'où les conséquences pour le fonctionnement de la station.

- inondations par surverse d'eau dans l'usine :

A partir de la cote de Seine de 25,63 NGF normal, cote d'arase des talus ceinturant l'usine, la station doit être protégée contre la surverse d'eaux pouvant arriver des terrains mitoyens à l'usine. Des digues sont établies pour protéger l'emprise de la station. Cette cote est supérieure à celle de la crue de 1910 (25,33 NGF normal à la station). Des tampons de regard sont également obturés.
En conclusion, une crue type 1910 aurait pour conséquence, en cas d'impossibilité de manœuvrer les vannes commandant l'arrivée des eaux à épurer ou de défaillance des relevages des eaux épurées (défaut d'alimentation EDF), de perturber et même d'arrêter le fonctionnement de la station d'épuration. Néanmoins, la réalisation d'alimentations complémentaires électriques devrait permettre d'écarter tout manque de courant. L'inondation de la station ne serait possible que pour une crue supérieure à celle de 1910 .

\subsection{Station d'épuration des eaux de Valenton}

La station de Valenton comprendra à terme deux tranches : la station 1, se décomposant en deux demi-tranches : Valenton 1A d'une capacité nominale de $150000 \mathrm{~m}^{3} /$ jour, et Valenton 1B d'une capacité nominale de $150000 \mathrm{~m}^{3 / \mathrm{j}}$, en service, et la station 2 en cours d'étude.

La capacité de traitement de cette deuxième tranche a été fixée à $300000 \mathrm{~m}^{3 /}$ jour par temps sec et $1200000 \mathrm{~m}^{3 /} / \mathrm{jour}$ par temps de pluie.

Le rejet des eaux épurées se fait par un canal de fuite vers la Seine. Il n'est pas prévu de station de relevage des eaux épurées en période de crue de la Seine.

La crue de 1910 a atteint la cote de 35,48 NGF normal au niveau du rejet de l'usine de Valenton.

La station risque d'être inondée par impossibilité de faire évacuer les eaux épurées dans les cas suivants :

- par temps sec, pour une crue de fréquence centennale qui atteindrait la cote de 34,72 NGF normal au niveau du rejet, c'est-à-dire de l'ordre de 7,90 m environ à l'échelle d'Austerlitz (33,47 usuelle Austerlitz) ;

- par temps de pluie, pour une crue de fréquence 10 à 20 ans, c'est-à-dire pour une hauteur comprise à Austerlitz entre $5,50 \mathrm{~m}$ et $6,40 \mathrm{~m}$ (soit entre 31,07 et 31,97 usuelle Austerlitz).

La résistance et la stabilité des décanteurs secondaires sont assurés jusqu'à un niveau maximum de la nappe de 33,50 NGF normal, soit 2 mètres environ inférieurs au niveau de la Seine pour une crue de type 1910.

\section{- 2.5 Station d'épuration des eaux de Colombes}

La nouvelle station d'épuration des eaux de Colombes qui aura une capacité de $240000 \mathrm{~m}^{3} / \mathrm{jour}$ est actuellement en cours de réalisation. Elle sera alimentée à partir de l'usine de Clichy.

La crue de 1910 a atteint à Colombes la cote de 28,73 NGF normal.

Lorsque la Seine atteint la cote 27,33 NGF normal au niveau de l'usine de Clichy, le canal de fuite vers la Seine est fermé. Les eaux sont évacuées gravitairement par l'émissaire Clichy-Achères, branche de Bezons, jusqu'à concurrence de $12 \mathrm{~m}^{3} / \mathrm{s}$. Au-delà de ce débit, les effluents sont repris par la station de pompage. Le départ vers l'émissaire général (c'està-dire vers Colombes) est fermé hormis un orifice permettant actuellement l'alimentation des installations du CRITER.

Donc, au-delà de la cote 27,33 NGF normal à Clichy, soit environ 26,33 NGF à Colombes (correspondant à une hauteur d'eau de 5 mètres à Austerlitz ( 30,57 usuelle Austerlitz environ), la future station d'épuration de Colombes n'assurera plus sa fonction épuratoire.

Les ouvrages de l'usine de Colombes sont protégés contre la surverse d'eau pouvant arriver des terrains mitoyens à l'usine (ou de la Seine elle-même) jusqu'à la cote de 29 NGF normal, soit à un niveau légèrement supérieur à celui de la crue de 1910 à ce site (28,73 NGF normal). 